\section{International Scientific Journal Theoretical \& Applied Science}

\author{
p-ISSN: 2308-4944 (print) e-ISSN: 2409-0085 (online) \\ $\begin{array}{lll}\text { Year: } 2016 \quad \text { Issue: } 7 & \text { Volume: } 39\end{array}$ \\ Published: $30.07 .2016 \quad \underline{\text { http://T-Science.org }}$
}

Mohiniso Bahromovna Hidirova Centre for the development of software and hardware program complexes at Tashkent University of Informational Technologies, Department of Regulatorika, Tashkent, Uzbekistan regulatorika@yahoo.com

SECTION 2. Applied mathematics. Mathematical modeling.

\title{
THEORETICAL BASES, METHODS AND TOOLKIT OF INFORMATION TECHNOLOGY «BIOREGULATORIKA»
}

\begin{abstract}
The article is deals with elaboration results concerning with theoretical bases, methods and hardware-software tools for bioregulatorika, received by "Regulatorika» group during 50 year. The given information technology assists in correction of organism condition in anomalies field for the purpose of moving into area with normal functioning. The bioregulatorika method is based on fundamental results on modeling and controlling living systems at norm and at anomalies taking into account time mutual relations in system of their regulation, processes cooperation and nonlinearity of biological feedback and is applied at an inefficiency of medicamentous treatment of the cancer and other diseases connected with regulatory mechanisms disorder.

Key words: chaos, cancer, mathematical modeling, nonlinearity, dynamical system, bio-feedback, regulatory mechanisms.

Language: Russian

Citation: Hidirova MB (2016) THEORETICAL BASES, METHODS AND TOOLKIT OF INFORMATION TECHNOLOGY «BIOREGULATORIKA». ISJ Theoretical \& Applied Science, 07 (39): 112-116.

Soi: http://s-o-i.org/1.1/TAS-07-39-20 Doi: crossef http://dx.doi.org/10.15863/TAS.2016.07.39.20
\end{abstract}

\section{ТЕОРЕТИЧЕСКИЕ ОСНОВЫ, МЕТОДЫ И ИНСТРУМЕНТАРИЙ ИНФОРМАЦИОННОЙ ТЕХНОЛОГИИ «БИОРЕГУЛЯТОРИКА»}

Аннотация: Данная статья посвящена результатам разработок теоретических основ, методов и
аппаратно-программных средств биорегуляторики, полученных в ходе более чем 50-летнего исследования
группой «Регуляторика». Данная информационная технология оказывает помошьь в коррекиии состояния
организма в области аномалий с иелью увода в область нормального функционирования. Метод
биорегуляторики основывается на фундаментальных результатах по моделированию и управлению живых
систем в норме и при аномалиях с учетом временных взаимоотношений в системе их регуляиии,
кооперативности процессов и нелинейности биологических обратных связей и применяется при
неэффективности медикаментозного лечения раковых и других заболеваний, связанных с нарушениями
регуляторных механизмов.

Ключевые слова: хаос, рак, математическое моделирование, нелинейность, динамические системы, биологическая обратная связь, регуляторные механизмы.

\section{Введение}

Проблемы сохранения и улучшения здоровья населения является актуальной задачей человечества в XXI веке. В статье приводятся результаты разработок теоретических основ, методов и программных средств количественных исследований функционирования регуляторных механизмов живых систем на молекулярногенетическом, клеточном и надклеточном уровнях организации и направленных на создание инструментария информационной технологии в биологии, медицине для обеспечения объективной, оперативной, экологически чистой и ресурсосберегающей технологией анализа механизмов саморегуляции живых систем, выявления эффективных точек воздействий в системе их регуляторики и оптимального управления функциональной активностью биосистем с целью достижения заранее заданных режимов их жизнедеятельности на основе метода математического моделирования регуляторики живых систем [1]. 


\begin{tabular}{|c|c|c|c|c|c|c|}
\hline Impact Factor: & $\begin{array}{l}\text { ISRA (India) } \\
\text { ISI (Dubai, UAE } \\
\text { GIF (Australia) } \\
\text { JIF }\end{array}$ & $\begin{array}{l}=1.344 \\
=0.829 \\
=0.564 \\
=1.500\end{array}$ & $\begin{array}{l}\text { SIS (USA) } \\
\text { PИНЦ (Russia) } \\
\text { ESJI (KZ) } \\
\text { SJIF (Morocco) }\end{array}$ & $\begin{array}{l}=0.912 \\
=0.234 \\
=1.042 \\
=2.031\end{array}$ & $\begin{array}{l}\text { ICV (Poland) } \\
\text { PIF (India) } \\
\text { IBI (India) }\end{array}$ & $\begin{array}{l}=6.630 \\
=1.940 \\
=4.260\end{array}$ \\
\hline
\end{tabular}

\section{Постановка задачи}

Математическое

моделирование регуляторных механизмов живых систем обычно предполагает количественный анализ поведения комплекса элементов, функционирующих в некоторой среде и способных реагировать на определенные внешние воздействия. Это приводит [1] к формулировке понятий or (операторов-регуляторов) регуляторной системы, способных к восприятию и синтезу сигналов определенной природы, - и asta (active system with time average) - сигнальной среды регуляторной системы, в которой взаимосвязанная деятельность элементов осуществляется, на основе обратных связей, с некоторым средним временем $h$ (временем, прошедшим с момента образования сигналов до момента воздействия их (или их продуктов) на активность элементов). Or вместе с asta cocтавляют регуляторную систему - orasta. Геометрия подобных регуляторных систем является динамической, в которой понятие неподвижной точки теряет смысл. Единицей времени orasta является $h$. Пусть имеем orasta c $n$ элементами. Уравнения такой системы, построенные с учетом кооперативности, временных взаимоотношений в asta и возможности, в некоторых случаях, сигналообразования в $\boldsymbol{a s t a}$ без участия or, имеют вид

$$
\begin{aligned}
& \frac{d x_{i}(t)}{d t}=\Lambda_{i}^{n}(X(t-h)) \exp \left(-\sum_{k=1}^{n} \delta_{i k} x_{k}\left(t-h_{i k}\right)\right)-b_{i} x_{i}(t) \\
& \text { где } \\
& \Lambda_{i}^{n}(X(t-h))=a_{i o}+\sum_{j=1}^{n}\left(\sum_{k_{1}, \ldots, k_{j}=1}^{n} a_{i k_{1}, \ldots, k_{j}} \prod_{m=1}^{j} x_{k_{m}}\left(t-h_{i k_{m}}\right)\right),
\end{aligned}
$$

с начальными условиями

$$
\begin{gathered}
x_{i}(t)=\varphi_{i}(t) n p u \quad t_{0}-h \leq t \leq t_{0}\left(t_{0}>h\right) ; \\
i, j, \quad k_{j}=1,2, \ldots, n,
\end{gathered}
$$

где $x_{i}(t)-$ величина, характеризующая количество сигнала, вырабатываемого $i$-ым or в момент времени $t ; h_{i k}-$ интервал времени, необходимого для изменения активности $i$-го or под действием активности $k$-го or; $a_{\text {iо }}, b_{i}-$ параметры скорости образования $i$-го сигнала в $\boldsymbol{a s t a}$, в or, распада $i$-го сигнала, соответственно; $\delta_{i k}$ - параметр репрессии $i$-го or продуктами деятельности $k$-го or.

Вектор $M_{c}\left(C_{l}, \ldots, C_{n}\right)$, значения элементов которого вычисляются по формулам

$$
C_{i}=\int_{0}^{\infty} \ldots \int_{0}^{\infty} \Lambda_{i}^{n}(S) \exp \left(-\sum_{j=1}^{n} \delta_{i k} S_{j}\right) d S_{1} \ldots d S_{n}-1
$$

является «мерой эволюции системы» и определяет возможные варианты развития, так как его величина, в случае конкретных систем, выделяет области возможных поведений на параметрическом портрете изучаемой системы. С другой стороны, $M_{c}$ выражает взаимоотношение системы регуляторики с внешней средой, поскольку его значение определяется заданными конкретными значениями коэффициентов. В случае $M_{c}=0$ система находится в равновесии с внешней средой.

Создание универсальной методологии анализа биорегуляторики систем организма на PC на основе математических моделей регуляторики живых систем и средств информационных технологий количественных исследований регуляторных механизмов живых систем на молекулярно-генетическом, клеточном и организменном уровнях организации позволит исследовать биосистему в норме и при различных заболеваниях оперативным, эффективным образом и корректировать состояние с целью достижения заранее заданных режимов их жизнедеятельности.

\section{Методы решения и обсуждение результатов}

Разработки основываются на более чем 50летнем опыте фундаментальных исследований по математическому моделированию биорегуляторики основных систем организма и прикладных исследований по разработке инструментария информационной технологии анализа регуляторных механизмов самоуправления биологических систем на основных уровнях организации (рисунок 1) [111].

Б.Н. Хидировым было введено понятие orasta, позволяющее разработать модельное обеспечение (в классе функциональнодифференциальных уравнений количественных исследований регуляторики живых систем с учетом пространственной разделенности, кооперативности, конкуренции за сигналы и комбинированной обратной связи биосистем. Были построены методы качественного и количественного анализа на РС характерных решений уравнений регуляторики биосистем с использованием фазовых и параметрических портретов, вычисления энтропии Колмогорова, показателя Ляпунова, Хаусдорфовой, информационной и высших размерностей. Методом качественного анализа было показано существование в системах регуляторики режимов покоя, устойчивого стационарного режима, автоколебаний, нерегулярных колебаний (динамический хаос) и режима резких 
деструктивных изменений - эффекта «черная дыра» (рисунок 2).

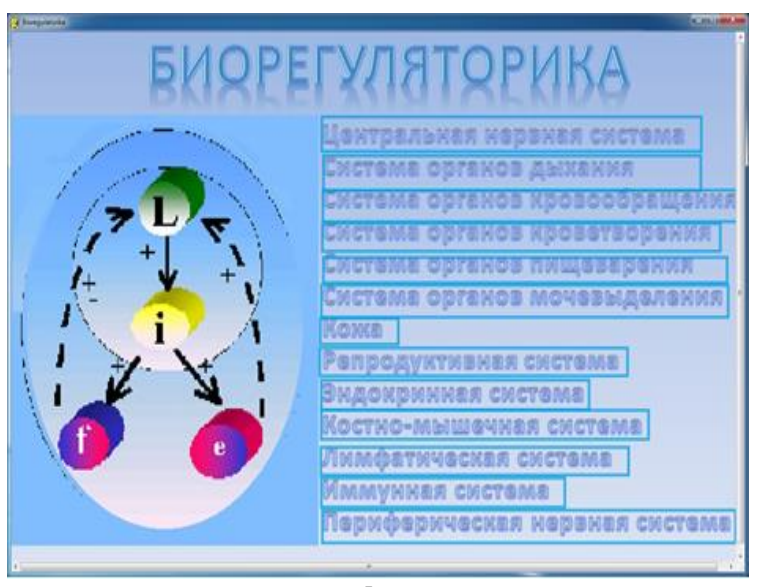

Рисунок 1 - Вид дисплея инструментария информационной технологии «Биорегуляторика».

Модельный анализ регуляторики конкретных систем организма позволил создать методику моделирования регуляторики основных функций: активности генов, биосинтетической активности клеток, митотического деления клеток, функций всасывания и секретообразования, кровообращения, кроветворения и других.

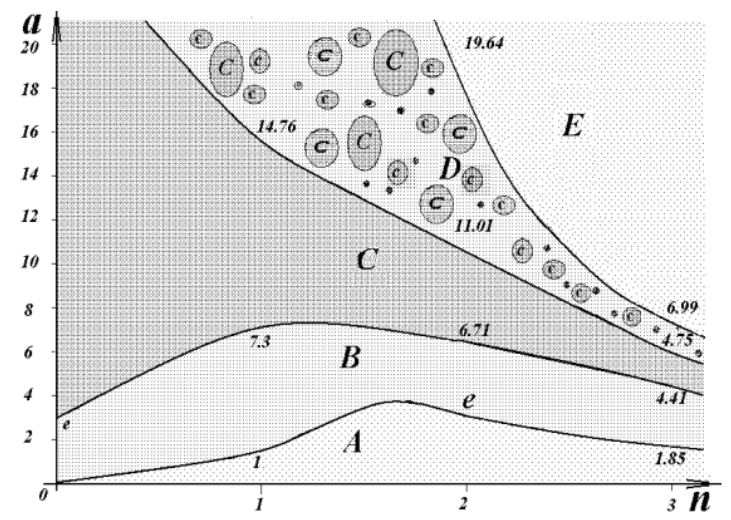

Рисунок 2 - Схематическое расположение областей параметрического портрета модельной системы

(1). (А- угасание, В - стационарный режим, C - автоколебания, D - динамический хаос, E деструктивные изменения - "черная дыра", (c) - малые регионы нормальной клеточной регуляторики (r-windows) в области динамического хаоса - D)

$\begin{array}{ccc}\text { Разработан } & \text { комплекс } & \text { программ, } \\ \text { осуществляющий } & \text { сопоставление } & \text { областей }\end{array}$ параметрических портретов модельных систем, которые строятся индивидуально для конкретной решаемой задачи с характерными показателями заболевания (вариабельность сердечного ритма, на основе электрокардиограмм сердечных заболеваний, показатели вирусной нагрузки, суточные колебания температуры и т.д.).

В рамках математического моделирования регуляторных механизмов живых систем актуальным является количественный анализ клеточной регуляторики в области $\mathrm{D}$ (рисунок 2). Динамика показателя Ляпунова, вычисленная на РC, позволяет оценивать хаотичность регуляторики и расположения малых регионов нормальной регуляторики (r-windows) в области D при различных значениях уровня интенсивности биологических процессов (рисунок 3). Наличие r-windows в области D свидетельствует о возможности управления клеточной регуляторикой в области D путем последовательного перемещения по сети rwindows с выходом в область устойчивых периодических изменений состояния клеточной регуляторики С (исунок 4).

Наиболее удобным способом улучшения состояния патологической системы является введение ее регуляторики в ближайший r-window путем малого воздействия. 


\begin{tabular}{l|lrl|l|ll} 
& ISRA (India) & $=\mathbf{1 . 3 4 4}$ & SIS (USA) & $=\mathbf{0 . 9 1 2}$ & ICV (Poland) & $=\mathbf{6 . 6 3 0}$ \\
Impact Factor: & ISI (Dubai, UAE) $=\mathbf{0 . 8 2 9}$ & PUHU (Russia) $=\mathbf{0 . 2 3 4}$ & PIF (India) & $=\mathbf{1 . 9 4 0}$ \\
& GIF (Australia) & $\mathbf{0 . 5 6 4}$ & ESJI (KZ) & $=\mathbf{1 . 0 4 2}$ & IBI (India) & $\mathbf{4 . 2 6 0}$
\end{tabular}

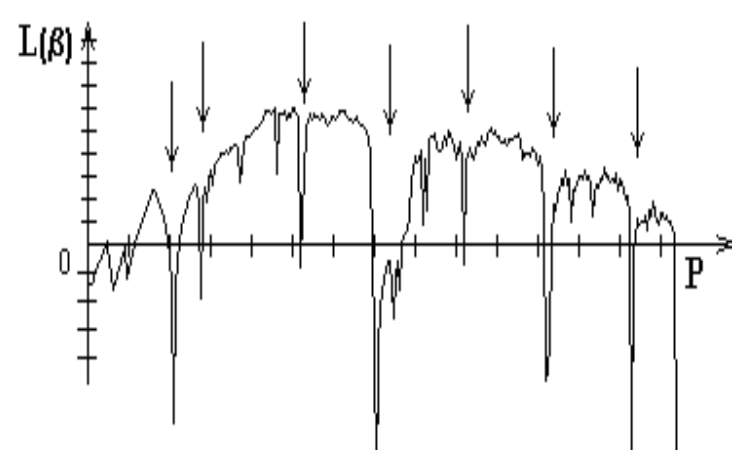

Рисунок 3 - График показателя Ляпунова L $(\beta)$ в D (стрелками указаны r-windows).

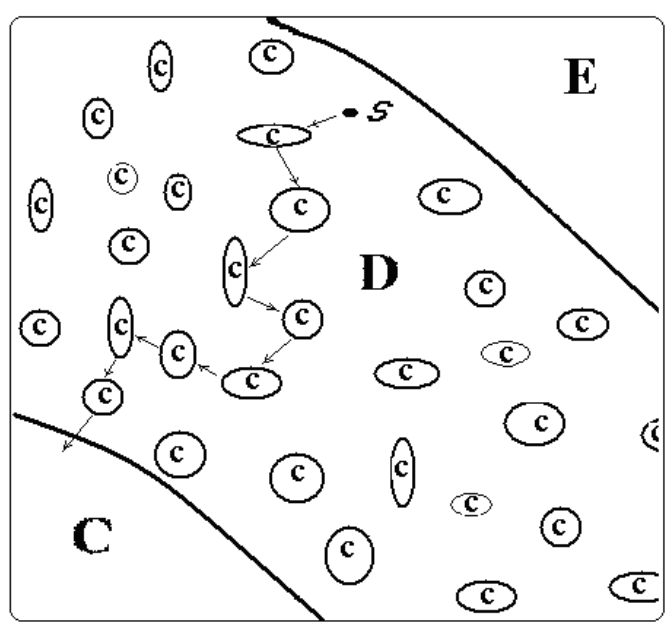

Рисунок 4 - Сценарий увода системы из области аномалий D в область нормы - C.

$$
L_{i}(t)=M_{i}(t)+C_{i}(t)+\sum_{k=1}^{N_{i}} R_{i k}(t)
$$

где $N_{i}$ число $\mathrm{r}$-windows на $i$-ом маршруте $(i=$ $1,2, \ldots, n)$.

В жизнедеятельности биологических систем эффект «черная дыра» встречается в виде крайней аномалии, ведущей к гибели системы. Примером может служить эффект внезапной остановки сердца, метастаза злокачественной опухоли, стадия увядания при вилте растений и т.д. Рассмотрим параметрический портрет на рисунке 2 для анализа возможных вариантов управления регуляторикой живых систем в области «черная дыра». Видно, что из области «черная дыра» Е возможен выход в область динамического хаоса D за счет уменьшения значений параметра $a$ и (или) параметра $n$. Регуляторика живых систем в области «черная дыра» может находиться в двух фазах: фазе нерегулярного колебания и в фазе коллапса с резким переходом решений в бассейн тривиального аттрактора. В фазе нерегулярных колебаний наиболее оптимальным уводом является последовательный переход по цепочке из r-windows в область динамического хаоса с использованием минимизации уровней нерегулярности и метаболической нагрузки. Во второй фазе (фазе резкого перехода решений в бассейн тривиального аттрактора) наиболее оптимальный увод системы в область динамического хаоса является переброска системы в первую фазу путем резкого снижения степени самосопряженности и (или) увеличения значения параметра активности системы регуляторики с дальнейшим решением задачи последовательного перехода в область С по цепочке из r-windows. В клинической практике наблюдаются эти варианты управления 
регуляторики живых систем. Например, в случае внезапной остановки сердца наблюдается отключение сознания (уменьшение $n$ ) и резкое уменьшение скорости всех физиологических функций организма (уменьшение $a$ ). Процедуры с электрическим воздействием при внезапной остановке сердца являются попыткой выброса системы сердечной регуляторики из второй фазы “черная дыра" в первую с нерегулярной ритмикой. Следует отметить необходимость оперативного проведения этих процедур, вследствие «обвального» разрушения динамической системы за очень короткий период времени.

\section{Заключение}

На основе параметрического портрета, настраиваемого индивидуально в ходе решаемой задачи, аппаратно-программный комплекс оказывает помощь в коррекции состояния организма в области аномалий с целью увода в область нормального функционирования на основе усовершенствования навыков саморегуляции. Метод регуляторики применяется при неэффективности применения лекарств в ходе лечении рака, группы заболеваний, связанных с нарушениями регуляторных механизмов. Главной целью метода регуляторики является немедикаментозное восстановление нормальной деятельности регуляторных систем организма, что приводит к устранению патологических симптомов и улучшению качества жизни. В перспективе планируется создание мобильных программно-аппаратных комплексов, персональных информационных технологий регуляторики конкретного организма с учетом нелинейных биологических обратных связей, саморегуляции, ингибирования конечным продуктом, ассоциативных взаимозависимостей.

\section{References:}

1. Hidirov BN (2014) Izbrannye raboty po matematicheskomu modelirovaniju reguljatoriki zhivyh sistem. Moscow - Izhevsk, 2014, 304 p.

2. Hidirov $\mathrm{BN}$ (1984) Ob odnom metode issledovanija reguljatoriki zhivyh sistem //Voprosy kibernetiki. - Tashkent, 1984. - Vyp. 128. - pp. 41-46.

3. Abduvaliev A, Saidalieva M, Hidirova M, Gildieva M (2015) Mathematical Modeling of the Thyroid Regulatory Mechanisms // American Journal of Medical Sciences and Medicine, 2015, Vol. 3, No 3, pp. 28-32.

4. Hidirov BN, Saidalieva M, Hidirova MB (1999) Modelirovanie reguljatoriki central'noj nervnoj sistemy i sistemy krovoobrashhenija //Algoritmy, - Tashkent. 1999, ANRUz. Vyp.87, - pp. 114-121.

5. Aliev BR, Hidirov BN, Saidalieva M, Hidirova M (2007) Quantitative Study of Cellular Mechanisms of HIV Infection's Pathogenesis //Engineering Letters, 2007. 13:3. pp. 304-307.

6. Hidirov BN, Aliev BR, Saidalieva M, Hidirova MB (2007) Modelirovanie i diagnostika reguljatoriki pecheni $\mathrm{v}$ norme $\mathrm{i}$ pri virusnyh gepatitah //Klinicheskaja medicina Kazahstana. - Kazahstan, 2007. - No 2(9). - pp. 13-15.

7. Saidalieva M, Aliev BR, Hidirov BN, Hidirova MB (2008) Perspektivy primenenija rezul'tatov matematicheskoj virusologii (na primere gepatita D) v klinicheskoj praktike //Problemy informatiki i jenergetiki. - Tashkent, 2008. -No 6. - pp. 27-32.

8. Gil'dieva AS, Hidirov BN, Saidalieva M (2009) Modelirovanie mehanizmov upravlenija chislennost'ju kletochnyh soobshhestv jepidermisa //Problemy informatiki i jenergetiki. - Tashkent, 2009, - No 3, pp. 14-19.

9. Saatov TS, Hidirov BN, Saidalieva M, Hidirova MB (2010) Matematicheskie modeli apoptoticheskogo upravlenija reguljatorikoj opuholevyh kletok //Problemy informatiki i jenergetiki. - Tashkent, 2010. - No 2. - pp.1217.

10. Hidirov BN, Saidalieva M, Hidirova MB (2007) Instrumentarij informacionnoj tehnologii upravlenija reguljatorikoj pecheni na kletochnom urovne (IITOT-1) //Gosudarstvennoe Patentnoe Vedomstvo RUz. No DGU 01411. 30.10.2007.

11. Hidirov BN, Saidalieva M, Hidirova MB (2008) Instrumentarij informacionnoj tehnologii upravlenija reguljatorikoj organov i tkanej (IITOT) //Gosudarstvennoe Patentnoe Vedomstvo RUz. No DGU 01596. 08.10.2008.

12. Saidalieva M, Hidirova M (2015) Computer System for Diagnostics of Disease Condition of an Organism Taking into Account the Basic Levels of Biosystem Organization // J. Biomath Communications Vol 2, No 1 (2015), pp. 528. 